$1-1-2021$

\title{
Towards automatic detection and quantification of mildew on grape leaf disks
}

\author{
Razib lqbal \\ Missouri State University \\ Kyle Sargent \\ MSU Graduate Student \\ László G. Kovács \\ Missouri State University
}

Follow this and additional works at: https://bearworks.missouristate.edu/articles-cnas

\section{Recommended Citation}

Iqbal, Razib, Kyle Sargent, and Laszlo Kovacs. 2021 "Towards Automatic Detection and Quantification of Mildew on Grape Leaf Disks." In Proceedings of the 18th International Conference on Signal Processing and Multimedia Applications, July 6 - July 8, 2021. https://doi.org/10.5220/0010583900810086.

This article or document was made available through BearWorks, the institutional repository of Missouri State University. The work contained in it may be protected by copyright and require permission of the copyright holder for reuse or redistribution.

For more information, please contact BearWorks@library.missouristate.edu. 


\title{
Towards Automatic Detection and Quantification of Mildew on Grape Leaf Disks
}

\author{
Razib Iqbal, Kyle Sargent and Laszlo Kovacs \\ College of Natural and Applied Sciences, Missouri State University, Springfield, MO, U.S.A.
}

Keywords: Background Removal, Downy Mildew, Grape Leaf, HSV Masking, Image Analysis.

Abstract: $\quad$ Downy and powdery mildews are the most serious diseases of the grapevine. A sustainable way to control these pathogens is the breeding and deployment of resistant grape cultivars. For breeding efforts to be effective, accurate quantification of the resistance phenotype is essential. In this paper, we present a computerbased image recognition, processing, and analysis technique for enhancing the detection and quantification of Plasmopara viticola and Erysiphe necator the causal agents of downy and powdery mildew, respectively. We propose a multi-step approach that utilizes background removal and Hue-Saturation-Value (HSV) masking as opposed to multi-faceted color channel breakdowns, photo texture evaluations, or classification-based algorithms for the detection of mildew. Our experimental results show that our method provides reliable results and fast performance.

\section{INTRODUCTION}

Plants can be classified based on two distinctions of infection, namely, non-infected (or normal) and infected (Awate et al, 2015). In the infected plants category, the growth of pathogen on plants is a major problem in the agricultural industry. To prevent it, many cultivators turn to harmful pesticides to slow/prevent the infection of it. While this practice is effective, it has many drawbacks. Instead, biologists have looked into breeding the plants selectively in order to breed samples that are naturally resistant to certain pathogens. In order to determine success in this manner, we need to analyze infected samples and determine the rate and amount of growth of infection on those samples. In this paper, we focus on grape leaf disks and the methods for detection and quantification of the mildew at both the microscopic level and human eye-level.

The existing methods for detecting mildew include color-space analysis, texture analysis, support vectors, and visual analysis (Awate et al, 2015; Sandika et al, 2016; Li et al, 2011; Vijayakumar, 2012). Hardware-based image analyses, such as (Cruz et al, 2016), rely on the capabilities of the hardware and the cost of the hardware is a factor in determining the aspects of the analysis. In comparison, visual analysis even though the most accessible and cost-efficient detection method has factors of bias from human perception. Its primary use is when quick and non-accurate readings are required to give a baseline for further analysis at a later point. Since this method is often accompanied by result variation, we have turned to computer-based image analysis for reliable and deterministic output that is useful to the end user.

Color space analysis can be further divided into multiple different categories, such as RGB colorspace analysis, BGR color-space analysis and HueSaturation-Value (HSV) color space analysis. As per (Vijayakumar, 2012), the RGB color-space can be split between the individual color channels to point out anomaly values caused by the growth of mildew. This method allows for a histogram approach, which accompanies calculating the mean value of each color channel and tracking changes in said values. HSV and BGR color spaces, also maintain the abilities from the RGB color-space analysis technique. However, creating a histogram of all colors in a single image can be very cumbersome on a machine depending on two factors: image quality and image resolution. Due to this, we elected to use color space masking to alleviate the necessity of histogram creation or any other expensive color channel tracking approaches. Our proposed approach tends to provide a reliable method for quantifying the mildew growth on grape leaves. 
The rest of the paper is organized as follows: In Section 2, we present our proposed approach with the implementation details. In Section 3, we present our performance evaluation. Finally, in Section 4, we conclude this paper with our observation followed by our future plans.

\section{PROPOSED APPROACH}

In order to measure the rate of mildew on the disk leaf samples we apply Background Removal (BR) and HSV masking to eliminate non-mildew spots from each photo samples. We then use the Laplacian of Gaussian (LoG) blob detection algorithm to quantify the amount of mildew contained in each photo. We explain these steps in the following subsections.

\subsection{Image Acquisition}

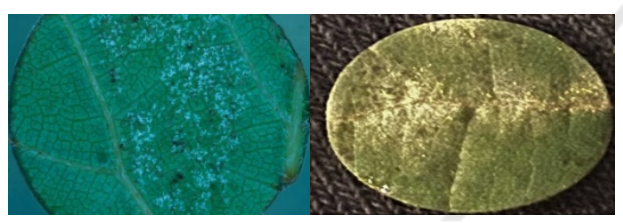

Figure 1: Photos of leaf disks infected with downy-and powdery-mildew at the micro- (left) and macroscopic-level (right).

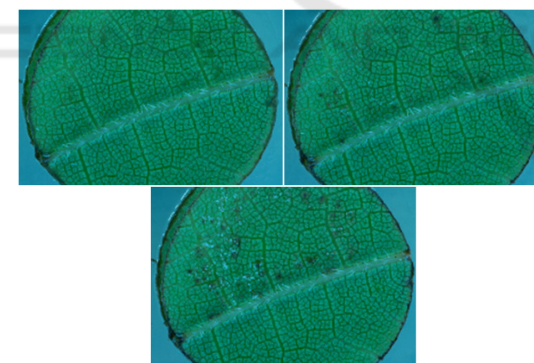

Figure 2: Single downy mildew-inoculated leaf disk over six-day span (left, right, bottom: 2dpi, 4dpi, 6dpi).

For our research, grape plants are cultivated as normal and given time to grow into mature vines. Once the plant has reached this stage, circular disks are cut from the plant's leaves, thus, supplying a grape leaf disk. These disks are then inoculated with $P$. viticola or E. necator as shown in Figure 1, and tracked over a six-day period. Throughout these six days, the growth of the mildew is tracked and photographs of the leaf disks at the macroscopic level are taken in the lab setting. On the sixth day, the resistance to the mildew is determined based on the extent of growth, which inversely related to disease resistance. During this process, visual analysis is conducted to deduce a baseline approximation of mildew on the leaf disk and resistance to the mildew.

The downy mildew images we used for this research were acquired from the laboratory of Dr. Lance Cadle-Davidson at the USDA Agricultural Research Service (Bierman et al, 2019). Our database included 53 photos in total with 48 of them being at the microscopic level and 5 being at the human eye level. The 43 microscopic photos span from 2-, 4-, and 6days past inoculation (dpi) of the leaf disk with the mildew and photos were taken under lab-grade microscopes and photo equipment with generally consistent lighting placement of leaf disks within the photos as shown in Figure 2. The macroscopic photos of powdery mildew infected leaf disks were taken with a USB camera with varying days past inoculation and varying distances from the disk under consistent lighting condition.

\subsection{Background Removal}

Background removal is the method in which the isolation of the leaf disk in the photo is done. This step is necessary because if the background has a similar color to that of the mildew, then false positive readings for mildew can occur. The steps for background removal are shown in Figure 3 and the respective outputs are shown in Figure 4.

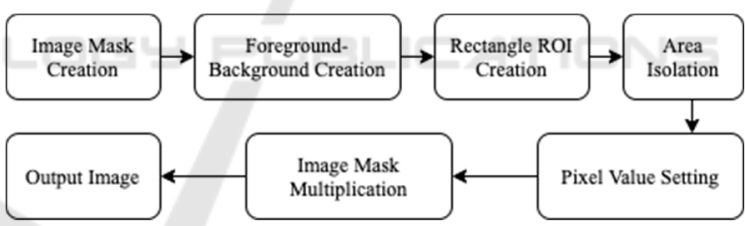

Figure 3: Steps for background removal.

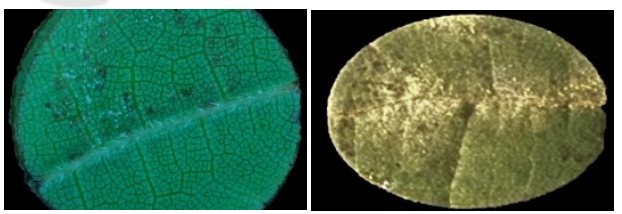

Figure 4: Background removed mirco- and macro- scopic level photos.

\subsection{HSV Masking}

HSV masking is the phase where the areas within the leaf disk that do not contain what is classified as mildew are masked out prior to blob detection and quantification. To accomplish this, an image's colorspace must be converted from BGR to HSV. The set of equations in (1) and (2) are used to convert 8/16bit images from BGR color space to HSV color space. 
Since BGR values can only lie between 0 and 255 , and HSV values lie between 0 and 255 for both Saturation and Value and 0-360 for Hue, an extra step of conversion is necessary to fit the Hue values between 0 and 255 for 8-bit images.

$$
\begin{gathered}
\mathrm{V} \rightarrow \max (\mathrm{R}, \mathrm{G}, \mathrm{B}) \\
\mathrm{S} \rightarrow\{\mathrm{V}-\min (\mathrm{R}, \mathrm{G}, \mathrm{B}) \quad \text { if } \mathrm{V} \neq 0 \\
0 \quad \text { otherwise }\} \\
\mathrm{H} \rightarrow\left\{\begin{array}{c}
60(\mathrm{G}-\mathrm{B}) /(\mathrm{V}-\min (\mathrm{R}, \mathrm{G}, \mathrm{B})) \text { if } \mathrm{V}=\mathrm{R} \\
120+60(\mathrm{~B}-\mathrm{R}) /(\mathrm{V}-\mathrm{min}(\mathrm{R}, \mathrm{G}, \mathrm{B})) \text { if } \mathrm{V}=\mathrm{G} \\
240+60(\mathrm{R}-\mathrm{G}) /(\mathrm{V}-\min (\mathrm{R}, \mathrm{G}, \mathrm{B})) \text { if } \mathrm{V}=\mathrm{B}\} \\
\mathrm{H} \rightarrow \mathrm{H} / 2
\end{array}\right.
\end{gathered}
$$

Since HSV is in three-dimensional space, two threshold arrays or vectors are created to specify a minimum and maximum threshold value. With this threshold, utilizing the third number, namely the value we want to mask over, allows us to keep only pixels whose value surpasses that of the minimum threshold. The steps to complete HSV masking are shown in Figure 5 and the respective output is presented in Figure 6.

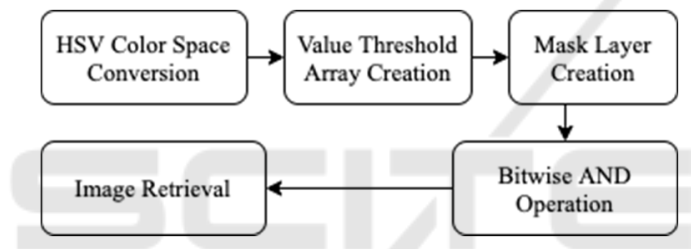

Figure 5: HSV masking steps.

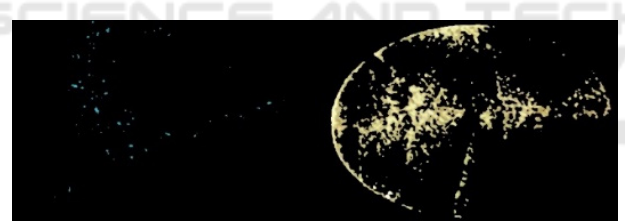

Figure 6: Output from HSV masking on micro- and macroscopic-level photos respectively.

\subsection{Blob Detection}

Blob detection is where the quantification of the mildew occurs. Once the masking has been completed, the resulting image that contains what is assumed to be mildew is once again converted to grayscale. The grayscale images shown in Figure 7 are computed by equation (3).

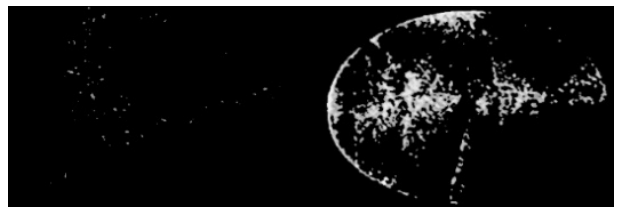

Figure 7: Grayscale version of sample photos after HSV masking.

$$
\begin{gathered}
\mathrm{Y} \rightarrow 0.299 \cdot \mathrm{R}+0.587 \cdot \mathrm{G}+0.114 \cdot \mathrm{B} \\
g(x, y, t)=(1 / 2 \pi t) \cdot \mathrm{e}^{-\left(x^{2}+y^{2}\right) / 2 t} \\
L(x, y ; t)=g(x, y, t) \cdot f(x, y)
\end{gathered}
$$

Once the grayscale image is returned, we then utilize Blob detection for quantification of the mildew. There are three types of Blob detection algorithms that we initially considered - Laplacian of Gaussian, Difference of Gaussians and Determinant of Hessian. We chose to use the Laplacian of Gaussian approach since it gave us the most accurate and fastest output of the three. We capture the different size blobs found throughout the image using the equations in (4)-(7).

Equation (4) is used to convolve the input image, which produces a third equation that dictates how one function is shaped by another. Equation (5) shows the scale space representation of the original image after the convolution. The scale space representation is essentially a representation of the photo, with a Gaussian filter on it that amplifies as the scale $t$ grows in number. Then we apply the Laplacian Operator (6) to (5) that determines the blobs of scale $t$ as per (Lindeberg, 2013)

$$
\mathbf{\Delta}^{2} L=L_{x x}+L_{y y}
$$

Following the application of (6) to (5), the Laplacian of Gaussian algorithm outputs the following:

$$
\text { detectedBlob }=(\mathrm{x}, \mathrm{y}, \sigma)
$$

Here, $(\mathrm{x}, \mathrm{y})$ are the coordinates of the blob and $\sigma$ is the standard deviation of the gaussian kernel which detected the blob. Now, the radius of a single blob is $\approx \sqrt{ } 2 \sigma$. At this point, we use equation (8) to obtain the radius of the blob.

$$
\begin{aligned}
& \mathrm{r}=\sigma \cdot \sqrt{ } 2 \\
& A=\pi \cdot \mathrm{r}^{2}
\end{aligned}
$$

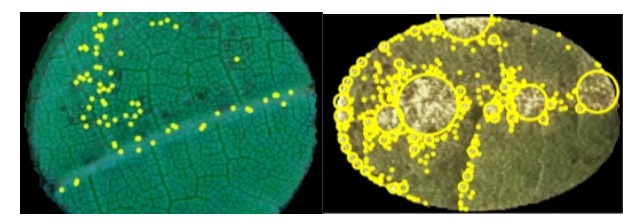

Figure 8: Detected blobs on micro- and macroscopic-level photos.

Now that we have the radius of said blob at coordinates $(\mathrm{x}, \mathrm{y})$, we find the area of a circle with said radius found by using (9). We can draw circles for $n$ blobs that were detected in the photo, as shown in Figure 8, and sum their areas together to retrieve the total area taken up by the blobs on a single leaf disk 
in pixel units. Once we retrieve all of the blob areas, we then use equation (11) to compute a leaf to mildew ratio.

TotalBlobArea $=\Sigma \mathrm{A}_{\mathrm{i}}$ (for $\mathrm{i}=0$ to $\left.\mathrm{i}=\mathrm{n}\right)$

TotalBlobArea/NonBlackPixelsAfterBR $\cdot 100$

\section{EVALUATION}

Table 1: Mildew growth over a 6-day period.

\begin{tabular}{|c|c|c|c|c|}
\hline $\begin{array}{c}\text { Tray } \\
\text { Number }\end{array}$ & $\begin{array}{c}\text { Photo } \\
\text { Number }\end{array}$ & $\begin{array}{c}\text { Mildew \% } \\
\text { at 2dpi }\end{array}$ & $\begin{array}{c}\text { Mildew \% at } \\
\text { 4dpi }\end{array}$ & $\begin{array}{c}\text { Mildew \% } \\
\text { at 6dpi }\end{array}$ \\
\hline 1 & 1 & 0.1193466 & 0.0 & 0.0055978 \\
\hline 2 & 1 & 0.9503586 & 0.0413495 & 0.0117653 \\
\hline 3 & 1 & 0.0061946 & 0.33576335 & 0.0369914 \\
\hline 4 & 1 & 0.3733049 & 0.3357633 & 0.3462468 \\
\hline 5 & 1 & 0.1799363 & 0.097927 & 0.1035048 \\
\hline 6 & 1 & 0.3605445 & 0.4817453 & 0.0835825 \\
\hline 7 & 1 & 0.0625684 & 0.0995543 & 0.092579 \\
\hline 8 & 1 & 0.0550063 & 0.0791701 & 0.175554 \\
\hline 1 & 2 & 1.6531951 & 1.3868999 & 2.173476 \\
\hline 2 & 2 & 0.5955877 & 0.5151023 & 0.3470667 \\
\hline
\end{tabular}

We tested our approach in two facets: growth tracking and quantification/detection. The growth tracking ability of our approach was tested by selecting a singular plant and tracking its mildew growth. This test was conducted on 10 of the 48 microscopic level images. For the data presented in Table I, we tested 10 different samples over a six-day span. From the reported results, we observed two trends, namely, decreasing and increasing. However, the other trend shown in the table, by rows 1 and 9 , is that of fluctuation. This trend is caused by the thresholding aspect of the approach and the appearance of cell structures that closely resemble the coloring of the mildew. Also, the mildew requires viable cells to live in, and therefore, if cells die then so does the mildew. This phenomenon causes fluctuation in the amount of mildew detected and quantified over the test period.

For quantification/detection evaluation, we tested on two different computers - a laptop with 8GB of RAM with an Intel Core i7 CPU @ 2.80GHZ processor and a desktop with $32 \mathrm{~GB}$ of RAM with an Intel Corei7 CPU @ 3.2GHz processor. We will refer to these two test machines as Machine A and Machine $\mathrm{B}$, respectively.
Table 2: Leaf to mildew ratios - Machine A.

\begin{tabular}{|c|c|c|c|c|}
\hline $\begin{array}{c}\text { Days Past } \\
\text { Inculation } \\
\text { (DPI) }\end{array}$ & Photo Date & $\begin{array}{c}\text { Tray } \\
\text { Number }\end{array}$ & $\begin{array}{c}\text { Photo } \\
\text { Number }\end{array}$ & $\begin{array}{c}\text { Mildew \% } \\
\text { (pixels) }\end{array}$ \\
\hline 2 & $9-15-18$ & 3 & 1 & 0.0 \\
\hline 6 & $9-19-18$ & 5 & 2 & 0.1533935 \\
\hline 6 & $9-19-18$ & 7 & 2 & 1.4873933 \\
\hline 6 & $9-19-18$ & 1 & 1 & 0.0059786 \\
\hline 6 & $9-19-18$ & 8 & 1 & 0.1755536 \\
\hline 6 & $9-19-18$ & 6 & 1 & 0.0835783 \\
\hline 4 & $9-17-18$ & 4 & 1 & 0.3357634 \\
\hline 4 & $9-17-18$ & 2 & 1 & 0.0413495 \\
\hline 2 & $9-15-18$ & 1 & 2 & 1.6531951 \\
\hline 2 & $9-15-18$ & 3 & 2 & 0.8965174 \\
\hline
\end{tabular}

Table 3: Leaf to mildew ratios - Machine B.

\begin{tabular}{|c|c|c|c|c|}
\hline $\begin{array}{c}\text { Days Past } \\
\text { Inoculation } \\
\text { (DPI) }\end{array}$ & Photo Date & $\begin{array}{c}\text { Tray } \\
\text { Number }\end{array}$ & $\begin{array}{c}\text { Photo } \\
\text { Number }\end{array}$ & $\begin{array}{c}\text { Mildew \% } \\
\text { (pixels) }\end{array}$ \\
\hline 4 & $9-17-18$ & 2 & 2 & 0.0059078 \\
\hline 2 & $9-15-18$ & 4 & 1 & 0.07149801 \\
\hline 2 & $9-15-18$ & 5 & 1 & 0.05584116 \\
\hline 6 & $9-19-18$ & 1 & 1 & 0.0 \\
\hline 6 & $9-19-18$ & 7 & 1 & 0.0555477 \\
\hline 6 & $9-19-18$ & 8 & 1 & 0.1271250 \\
\hline 4 & $9-17-18$ & 3 & 1 & 0.0651918 \\
\hline 4 & $9-17-18$ & 6 & 1 & 0.2470763 \\
\hline 2 & $9-15-18$ & 6 & 2 & 0.0609538 \\
\hline 2 & $9-15-18$ & 8 & 2 & 0.4067885 \\
\hline
\end{tabular}

We tested the system with the following tests: 1 photo, 2 photos, 3 photos, 4 photos, up to 8 photos at a time. Since we tested a total of 36 photos per test for Tables II and III, we display only the first 10 results without losing any important information. In Table II, we showcase the quantification results from Machine $\mathrm{A}$, and in Table III, we showcase the results from Machine B. Photos were chosen at random from three date folders of 9-15-18, 9-17-18 and 9-19-18 and then from one of the 8 tray folders within each date. The photos selected for this test were also solely at the microscopic level since the macroscopic-level photos 
did not belong to a specific date folder. The mildew percentages are in terms of pixel which is calculated by dividing the number of pixels that existed after removing the background by the number of pixels the blobs of mildew took up. Higher density of mildew produces a higher mildew percentage overall. Since these numbers are computer generated, they were same for both machines, which is why we chose the photos at random for both machines. Also, for the two machines, there was a difference in the HSV mask threshold values. The $8 \mathrm{~GB}$ machine ran with a threshold value of 170 and the $32 \mathrm{~GB}$ machine ran with a threshold of 180 . This explains the difference in the outputs of row 4, column 5 in both Tables II and III.

Table 4: Detection-Quantification runtimes - Machine A.

\begin{tabular}{|c|c|c|c|c|}
\hline $\begin{array}{c}\text { \# of } \\
\text { Tested } \\
\text { Photos }\end{array}$ & $\begin{array}{c}\text { Avg. } \\
\text { Detection } \\
\text { Runtime } \\
(\mathrm{ms})\end{array}$ & $\begin{array}{c}\text { Total } \\
\text { Detection } \\
\text { Runtime } \\
(\mathrm{ms})\end{array}$ & $\begin{array}{c}\text { Avg. } \\
\text { Quantification } \\
\text { Runtime (ms) }\end{array}$ & $\begin{array}{c}\text { Total } \\
\text { Quantification } \\
\text { Runtime (ms) }\end{array}$ \\
\hline 1 & 1.3819 & 1.3819 & 374.1365 & 374.1365 \\
\hline 2 & 5.85395 & 11.70789 & 498.7908 & 997.5817 \\
\hline 3 & 129.16577 & 387.4973 & 649.2774 & 1947.83229 \\
\hline 4 & 104.20022 & 416.80089 & 976.5763 & 3906.30529 \\
\hline 5 & 14.38234 & 71.91169 & 823.5583 & 4117.79159 \\
\hline 6 & 40.49657 & 249.7939 & 958.8845 & 5753.307709 \\
\hline 7 & 53.75931 & 376.151 & 1031.3986 & 7219.79089 \\
\hline 8 & 60.03296 & 480.2636 & 1451.8371 & 11614.6974 \\
\hline
\end{tabular}

Table 5: Detection-Quantification runtimes - Machine B.

\begin{tabular}{|c|c|c|c|c|}
\hline $\begin{array}{c}\text { \# of } \\
\text { Tested } \\
\text { Photos }\end{array}$ & $\begin{array}{c}\text { Avg. } \\
\text { Detection } \\
\text { Runtime } \\
(\mathrm{ms})\end{array}$ & $\begin{array}{c}\text { Total } \\
\text { Detection } \\
\text { Runtime } \\
(\mathrm{ms})\end{array}$ & $\begin{array}{c}\text { Avg. } \\
\text { Quantification } \\
\text { Runtime (ms) }\end{array}$ & $\begin{array}{c}\text { Total } \\
\text { Quantification } \\
\text { Runtime (ms) }\end{array}$ \\
\hline 1 & 8.4423 & 8.4423 & 274.828 & 274.828 \\
\hline 2 & 4.9833 & 9.9665 & 371.36 & 742.721 \\
\hline 3 & 79.1446 & 237.4338 & 349.585 & 1048.750 \\
\hline 4 & 68.0872 & 272.3490 & 646.065 & 2584.260 \\
\hline 5 & 66.904 & 334.5221 & 1062.229 & 5311.147 \\
\hline 6 & 59.927 & 359.5607 & 1151.012 & 6906.069 \\
\hline 7 & 13.547 & 94.8268 & 1050.920 & 7356.4422 \\
\hline 8 & 76.119 & 608.9588 & 1416.313 & 11330.505 \\
\hline
\end{tabular}

Table IV and Table V showcase the detectionquantification run-time performance for 8 different tests. As expected, we see a trend in which as the numbers increase, the total and average times increase as well. However, depending on the amount of quantification or detection time that one photo takes depend on the amount of mildew that resides within the photo itself. Again, as with the mildew percentages found in Tables II and III, less resistant plants will take a longer time to detect and quantify the mildew that resides on the leaf disks.

\section{CONCLUSIONS}

In this paper, we proposed a multi-step approach for the detection and quantification of mildew diseases that reside on either the top or the bottom of grape leaves. We are working towards a fully automated detection process to perform quantitative analysis of the mildew growth in an outdoor setting. It will add value to the process of selectively breeding grapes based on their resistance.

The approach presented in this paper contains the ability to detect and quantify mildew at both the microscopic and macroscopic level. To acclimate to the issue of thresholding, we used a number between 170 to 180 to retrieve the most optimal results. Any value less than 170 for the set of photos we used allowed for more cell structures and anomalies within the photo, e.g., lens flare from the camera and microscope, to be captured and identified as mildew as well. Therefore, we recommend that a threshold number between 170 and 180 be used to eliminate majority of the false positives that could occur during detection.

We also observed that the utilization of a blur while thresholding allowed for more accurate results. Because of the intricacies of the cells, the blue helped to soften some of the cell pixels that could produce false positives. To accomplish this, a gaussian blur of 3 was applied on the photos and anything higher causes pixels to become too blurry for the blob detection algorithm to pick them up correctly.

In the future, we plan to continue adjusting threshold values and begin testing the approach with more than just grape leaf disks to determine if the approach can successfully capture mildew diseases of other kinds that may grow on other plants as well. An example could be, looking at the powdery mildew, known as Podosphaera leucotricha, that grows on apples. 


\section{REFERENCES}

Awate, A., Deshmankar, D., Amrutkar, G., Bagul, U., \& Sonavane, S. (2015). Fruit disease detection using color, texture analysis and ANN. 2015 International Conference on Green Computing and Internet of Things (ICGCIoT), pp. 970-975.

Bierman, A., LaPlumm, T., Cadle-Davidson, L., Gadoury, D., Martinez, D., Sapkota, S., \& Rea, M. (2019). A high-throughput phenotyping system using machine vision to quantify severity of grapevine powdery mildew. Plant Phenomics.

Cruz, J. A., Yin, X., Liu, X., Imran, S. M., Morris, D. D., Kramer, D. M., \& Chen, J. (2016). Multi-modality imagery database for plant phenotyping. Machine Vision and Applications, 27(5), pp. 735-749.

Li, G., Ma, Z., \& Wang, H. (2011). Image recognition of grape downy mildew and grape powdery mildew based on support vector machine. International Conference on Computer and Computing Technologies in Agriculture, pp. 151-162.

Lindeberg, T. (2013). Scale-space theory in computer vision (Vol. 256). Springer Science \& Business Media.

Sandika, B., Avil, S., Sanat, S., \& Srinivasu, P. (2016). Random forest based classification of diseases in grapes from images captured in uncontrolled environments. 2016 IEEE 13th International Conference on Signal Processing (ICSP), pp. 1775-1780.

Vijayakumar, J., \& Arumugam, S. (2012). Recognition of powdery mildew disease for betelvine plants using digital image processing. International Journal of Distributed and Parallel Systems, 3(2), 231. 